9-1-2016

Management and outcomes of intramedullary spinal cord tumors:

A single center experience from a developing country

Dr Saqib Kamran Bakhshi

Muhammad Waqas

Baila Shakaib

Ather Enam

Follow this and additional works at: https://ecommons.aku.edu/pakistan_fhs_mc_surg_neurosurg

Part of the Neurology Commons, Neurosurgery Commons, and the Surgery Commons 


\title{
Management and outcomes of intramedullary spinal cord tumors: A single center experience from a developing country
}

\author{
Saqib K. Bakhshi, Muhammad Waqas, Baila Shakaib, Syed A. Enam \\ Department of Surgery, Section of Neurosurgery, Aga Khan University Hospital, Karachi, Pakistan \\ E-mail: Saqib K. Bakhshi - saqib_dmc@hotmail.com; MuhammadWaqas - shaiq_waqas@hotmail.com; Baila Shakaib - bailashakaib@gmail.com; \\ *Syed A. Enam - ather.enam@aku.edu \\ *Corresponding author \\ Received:01 July 16 Accepted: 13 July 16 Published:01 September 16
}

\begin{abstract}
Background: Intraoperative neurophysiology, high magnification microscopes, and ultrasonic aspirators are considered essential aid for the safe resection of intramedullary spinal cord tumors (IMSCTs). Most centers in developing countries such as Pakistan still lack these facilities. The purpose of this study was to review the management of IMSCTs at our hospital and to determine factors associated with the outcomes of surgery.
\end{abstract}

Methods: This was a retrospective review of medical records of adult patients undergoing surgery for IMSCT over 12 years. The institutional ethical review committee approved this study. Data were collected regarding demographics, clinical and radiological features, and surgical details. Modified McCormick Scale was used to grade patients' neurological status at admission, discharge, and follow-up. Statistical analysis was performed using the Statistical Package for Social Sciences version 22.

Results: Forty three cases were reviewed. Mean age was $33.8 \pm 15.1$ years whereas median follow-up was 5 months (range: $0.25-96$ months). Most patients had ependymoma $(n=16 ; 73 \%)$. Cervical region was the most commonly involved $(n=15 ; 34.9 \%)$. Gross total resection (GTR) was achieved in 30 cases $(69.8 \%)$. The preoperative McCormick grade was significantly associated with follow-up McCormick grade $(P$ value $=0.002)$. Eight patients $(18.6 \%)$ underwent intraoperative electrophysiological monitoring, out of which GTR was achieved in all cases, and none had disease progression or recurrence. Ten patients received postoperative radiotherapy. Thirty five patients $(81.4 \%)$ had progression free survival at last follow-up.

Conclusions: We achieved a GTR rate of $68.9 \%$ for IMSCTs with limited resources. In few cases, where intraoperative electrophysiology was used, the rate of GTR was $100 \%$. Preoperative neurological status was associated with better postoperative McCormick score.

Key Words: Intramedullary spinal cord tumor, modified McCormick scale, spinal cord

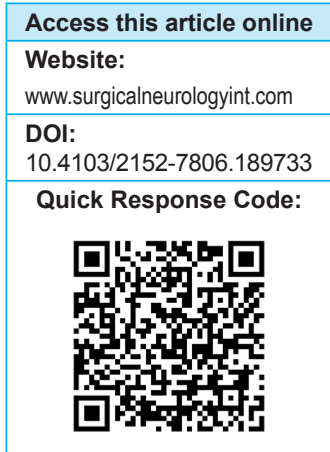

This is an open access article distributed under the terms of the Creative Commons Attribution-NonCommercial-ShareAlike 3.0 License, which allows others to remix, tweak, and build upon the work non-commercially, as long as the author is credited and the new creations are licensed under the identical terms.

For reprints contact: reprints@medknow.com

How to cite this article: Bakhshi SK, Waqas M, Shakaib B, Enam SA. Management and outcomes of intramedullary spinal cord tumors:A single center experience from a developing country. Surg Neurol Int 2016;7:S617-22.

http://surgicalneurologyint.com/Management-and-outcomes-of-intramedullary-spinal-cord-tumors:-A-single-center-experience-from-a-developing-country/ 


\section{INTRODUCTION}

Intramedullary spinal cord tumors (IMSCT) account for only $4-10 \%$ of all primary central nervous system (CNS) neoplastic lesions and $20 \%$ of all spinal cord tumors in adults. ${ }^{[8]}$ Although uncommon, IMSCTs can cause disability and severely affect the quality of life. ${ }^{[8,11]}$ Spinal ependymoma is the most frequently occurring IMSCT. ${ }^{[8,11]}$ Magnetic resonance imaging (MRI) is the imaging modality of choice for the diagnosis and characterization of these lesions. ${ }^{[20]}$

According to previous studies regarding treatment options, early surgical excision, possibly gross total resection (GTR), improves functional outcomes. ${ }^{[11,16,18]}$ Numerous authors have endorsed regular use of intraoperative electrophysiological monitoring (IOEM) including somatosensory-evoked potentials (SSEP) and motor-evoked potentials (MEP); however, there is insufficient evidence assessing their real impact on functional outcomes. . $^{[6,9,14,17]}$

There is scarcity of data on IMSCT from Pakistan ${ }^{[1]}$ and other countries from South Asia, where performing maximum resection of tumor becomes even more challenging due to resource limitations. The purpose of this study was to review the presentation, management, and outcomes IMSCTs at our hospital during last 12 years and to determine the factors associated with outcomes of surgery.

\section{MATERIALS AND METHODS}

This was a retrospective review. All patients who underwent surgery for IMSCT at our hospital between 2003 and 2015 with complete records available, irrespective of age and gender, were included. The patients were identified by the Medical Records department of the hospital, which then retrieved their medical records. Ethical approval was obtained from the Ethical Review Committee at Aga Khan University Hospital, Karachi (3533-Sur-ERC-15).

Data was collected according to a proforma. It consisted of different variables including demographics, clinical presentation of disease and management in the hospital, histopathological diagnosis, and postoperative course of the disease. This information was extracted by reviewing patients' medical records. Patients with incomplete records were excluded from the study.

GTR was defined as the removal of $>95 \%$ tumor tissue, which was either mentioned in operative notes or seen on postoperative MRI. Maximum safe resection (MSR) was defined as the removal of maximum volume of tissue, which could be safely removed. Biopsy was defined as the removal of only a small amount of tissue required for histological analysis.
Use of IOEM is not a standard practice at our hospital so it was utilized in some cases using SSEP. Tumor was histologically diagnosed and then graded according to the World Health Organization (WHO) classification at the histopathology department of our hospital. Progression free survival (PFS) was defined as no recurrence of disease after GTR and no increase in the size of residual disease after MSR or biopsy.

Modified McCormick Scale (MMS) was used for grading patients' neurological status at admission, immediately after surgery and at last follow-up [Table 1]. ${ }^{[13]}$

\section{Surgical technique for intramedullary spinal} cord tumors

We perform the procedure in prone position under general anesthesia. For cervical surgeries, Mayfield clamp is used to fix the head in a flexed position. It is ensured that abdomen is free and all the pressure points are adequately padded. We confirm the level of surgery with the help of fluoroscope before starting incision and after exposure of laminae. For cases where intraoperative electrophysiology monitoring is planned, electrodes are placed after positioning. A midline posterior approach is used with subperiosteal dissection to expose laminae bilaterally. Facet joints are carefully preserved. Laminectomy is performed at the level of the tumor, ensuring that we have an adequate exposure of cranial and caudal limits of the tumor. In few cases, we have also performed laminoplasty without compromising exposure. After extradural hemostasis, a midline durotomy is performed using hook and knife. Posterior median sulcus is then identified and gently opened to access the tumor. We do not use coagulation and stay within the limits of the tumor performing internal debulking with the help of dissector and tumor forceps. After limited debulking, we dissect the tumor from margins and roll it inward.

Once the dissection is completed till the normal cord we complete the hemostasis. We use surgical (fibrillar and other) instead of cotton balls. Our use of cavitron ultrasonic surgical aspirator (CUSA) is very limited because of the high cost associated with its disposables. SSEP monitoring is not used consistently. Dura is closed in a watertight fashion using nonabsorbable suture. Valsalva maneuver is performed to look for any leak. Wound is then closed in layers.

Table 1: Modified McCormick scale

\begin{tabular}{ll}
\hline Grade & Modified McCormick Scale \\
\hline I & Intact neurologically, normal ambulation, minimal dysesthesia \\
II & Mild motor or sensory deficit, functional independence \\
III & Moderate deficit, limitation of function, independent with \\
& $\begin{array}{l}\text { external aid } \\
\text { IV }\end{array}$ \\
V & Severe motor or sensory deficit, limited function, dependent \\
\hline
\end{tabular}




\section{Statistical analysis}

Statistical Analysis was done using Statistical Package for Social Sciences (SPSS Inc., Chicago, IL, USA. Version 22.0). Means and standard deviation were calculated for continuous data with normal distribution whereas median and interquartile range (IQR) were calculated for continuous data with skewed distribution. Percentages and proportions were calculated for categorical data. Fisher's exact test was used to compare categorical data. Preoperative neurological status, tumor histology, extent of resection, and IOEM were correlated with postoperative neurological outcome. Kaplan-Meier graph was plotted to portray PFS.

\section{RESULTS}

Forty three patients were included in the study. Median age was 33.2 years, and the most common presenting complaint was back pain present in 24 patients (55.8\%). The most common location was cervical spine $(n=15$; 34.9) Only 3 patients (7.0\%) had single level disease and half of the patients had their disease involving 2 or 3 spinal levels $(n=22 ; 51.1 \%)$. The highest numbers of disease levels involved were 7 present in $2(4.7 \%)$ patients. The most common histological diagnosis was ependymoma. Patients' demographics and clinical characteristics are shown in Table 2.

We performed GTR in 30 patients (69.8\%). Intraoperative electrophysiological monitoring (IOEM) was used in $8(18.6 \%)$ patients and GTR was achieved in all of them. Twenty nine (67.4\%) patients had one or more postoperative MRI done. Ten patients (23.3\%) received postoperative radiotherapy while none of the patients received postoperative chemotherapy.

\section{Neurological status}

Postoperatively, 15 patients (34.9\%) showed improvement in their neurological function before discharge, $10(23.2 \%)$ had transient worsening of function, and 18 (41.9\%) had no change. At last follow-up, an improvement in neurological function was observed in 24 patients $(55.8 \%), 15(34.9 \%)$ maintained their preoperative function, and only 4 patients $(9.3 \%)$ had persistently worsened neurological status. Graph 1 shows that better pre-operative MMS grades that resulted in better neurological function postoperatively $(P=<0.001)$ and at last follow-up $(P=0.002)$ [Graph 1].

Graph 2 depicts change in neurological status at last follow-up compared to the preoperative status, and the role of IOEM, which was not statistically significant $(P=0.456)$ [Graph 2].

Progression free survival

Eight patients (18.6\%) had recurrence or progression of disease after surgery. Only $3 \%$ of all the patients who had GTR had disease progression, whereas $45 \%$ patients with MSR and all patients with biopsy had disease progression $(P=0.004)$. Thirty five patients $(81.4 \%)$ had progression free survival at last follow-up. Table 3 shows some possible predictors of PFS.

Patients undergoing GTR had a significantly better survival as compared to those who underwent MSR or only biopsy on Kaplan-Meier log-rank analysis

Table 2: Demographics and clinical characteristics

\begin{tabular}{ll}
\hline \multicolumn{2}{l}{ Demographics and Clinical Characteristics } \\
\hline Age (Median) & 33.2 years (IQR=24-46 years) \\
Gender & \\
Male & $n=27(62.8 \%)$ \\
Female & $n=16(37.8 \%$ \\
Comorbids & \\
Hypertension & $n=6(14.0 \%)$ \\
Diabetes mellitus & $n=2(4.6 \%)$ \\
Ischemic heart disease & $n=1(2.3 \%)$ \\
Benign prostatic hyperplasia & $n=1(2.3 \%)$ \\
Length of hospital stay (Median) & 7 days (IOR=4-10 days) \\
Follow-up time (Median) & 5 months (IOR=2-15 months) \\
Most common presenting complaint & Backache \\
Duration of symptoms (Median) & 4 months (IOR=2-12 months) \\
Location & \\
Cervical & $n=15(34.9 \%)$ \\
Thoracic & $n=9(20.9 \%)$ \\
Lumbar & $n=9(20.9 \%)$ \\
Cervicothoracic & $n=4(9.3 \%)$ \\
Thoracolumbar & $n=6(14.0 \%)$ \\
Operating time (Mean) & $n=30(69.8 \%)$ \\
Commonest histological type of & Ependymoma \\
tumor & $n=11(25.6 \%)$ \\
Histology & \\
Ependymoma & $n=26(60 \%)$ \\
Astrocytoma & $n=9(21 \%)$ \\
Oligodendroglioma & $n=2(5 \%)$ \\
Hemangioblastoma & \\
Ganglioglioma & \\
Extent of Resection & \\
\hline
\end{tabular}

Table 3: Predictors of progression free survival

\begin{tabular}{lc}
\hline \multicolumn{2}{c}{ Predictors of Progression Free Survival } \\
\hline Factors & $\boldsymbol{P}$ value \\
\hline Age & 0.443 \\
Gender & 0.411 \\
Size of Lesion (>3 levels) & 0.050 \\
IOEM & 0.679 \\
GTR & 0.002 \\
Low grade lesion (Grade I/II) & 0.011
\end{tabular}


$(P=<0.001)$, as shown in Figure 1. Similarly patients who had lower histopathological grade (grade I/II) had a better survival compared to those who had higher grades of lesion (grade III/IV), as shown in Figure 2.

\section{DISCUSSION}

Lesions involving the spinal cord have always tested surgeons' skill. Figure 3 (a-c) shows MRI scans of a 20-year-old girl who presented with progressive weakness in all four limbs, more on the right side, for 2 years. Her preoperative MMS was 3. She underwent cervical laminectomy and gross total excision of the lesion 5 years back. Her immediate postoperative MMS was 2, and at last follow-up after 4 years, it was 1. Last follow-up scans done 4 years after surgery are shown in Figure 4 (a-c).

Last few years have seen major advances toward better treatment of IMSCTs owing to developments in imaging techniques and surgical procedures. ${ }^{[9,11,12]}$ We studied patients with IMSCT who were surgically treated at

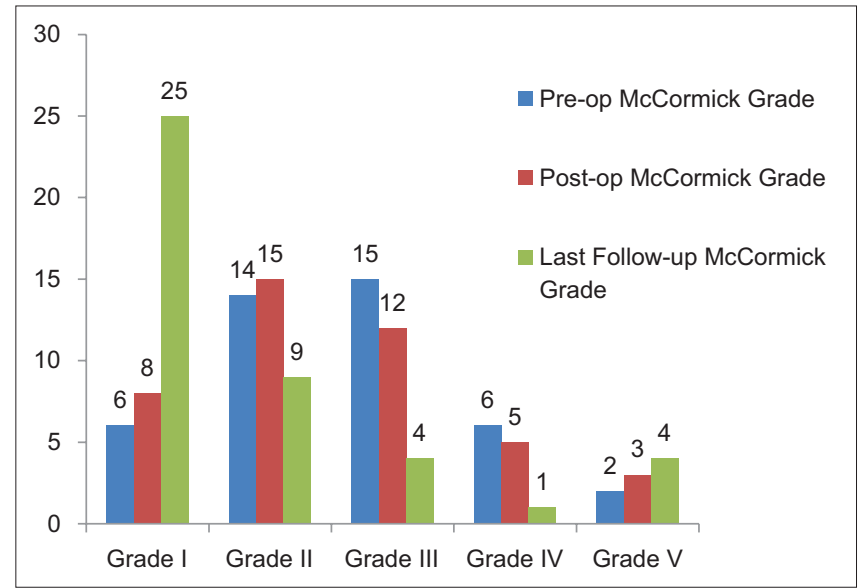

Graph I: McCormick grades on pre-operative, post-operative and last follow up examination

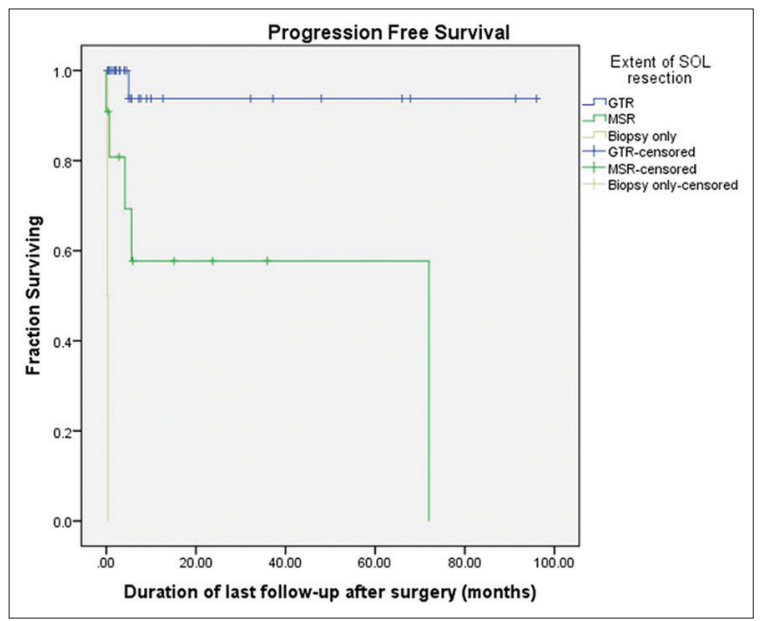

Figure I: Kaplan-Meier analysis for progression free survival with gross resection rate, maximum safe resection, and biopsy our hospital during last the 12 years; our sample of 43 patients is slightly less but comparable to most other studies, although few studies with larger sample size have been reported. ${ }^{[15,18]}$ Our median follow-up of 5 months is, however, less than previous studies. ${ }^{[15,18]}$

Surgeons operating on IMSCTs are faced with the predicament of attempting radical excision, and yet, preserve adequate neural tissue. In a retrospective review conducted in 2005 involving 78 patients, Sandalcioglu et al. ${ }^{[18]}$ reported that they achieved GTR in $83.3 \%$ patients and cited preoperative neurological status as the most important predicting factor of functional outcome. Similar findings were quoted by Kumar et al. ${ }^{[10]}$ in their study on 43 patients. In another series of 70 cases, Bostrom et al..$^{[2]}$ also reported complete removal in $64.3 \%$ cases and stated early GTR of spinal ependymoma to be associated with better outcomes, limiting role of MSR, or biopsy with adjuvant therapy for high grade astrocytoma. We achieved GTR in $69.7 \%$ patients and have also found statistically significant relationship between preoperative and last follow-up neurological function. Because of less number of cases, subgroup analysis for individual tumor types could not be done.

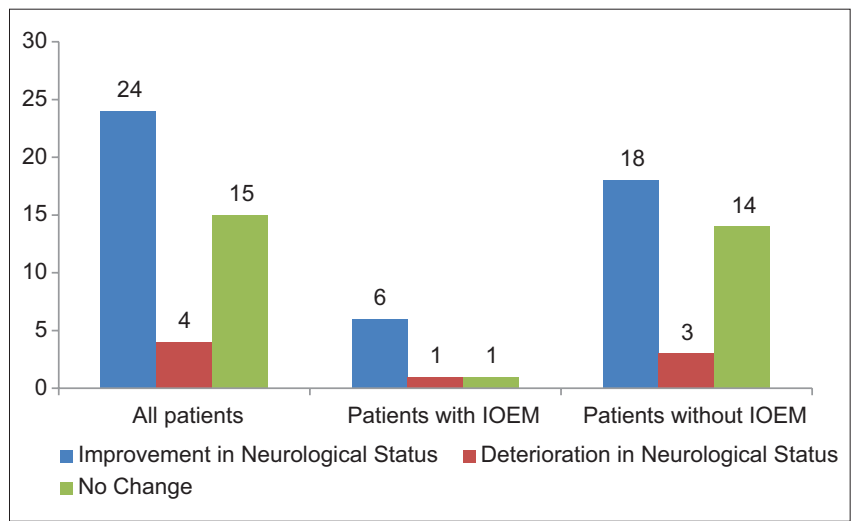

Graph 2: Change in neurological status at last follow-up and intraoperative electrophysiological monitoring

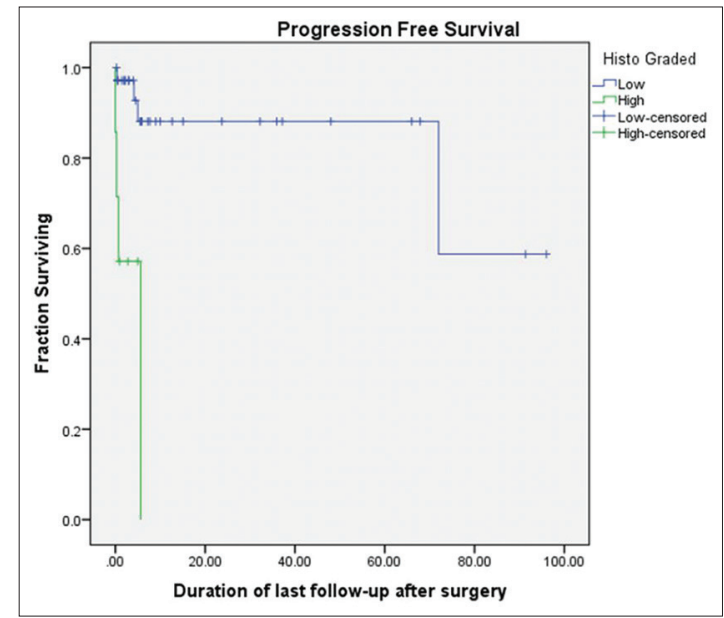

Figure 2: Kaplan-Meier analysis for progression free survival with regards to grade of lesion 

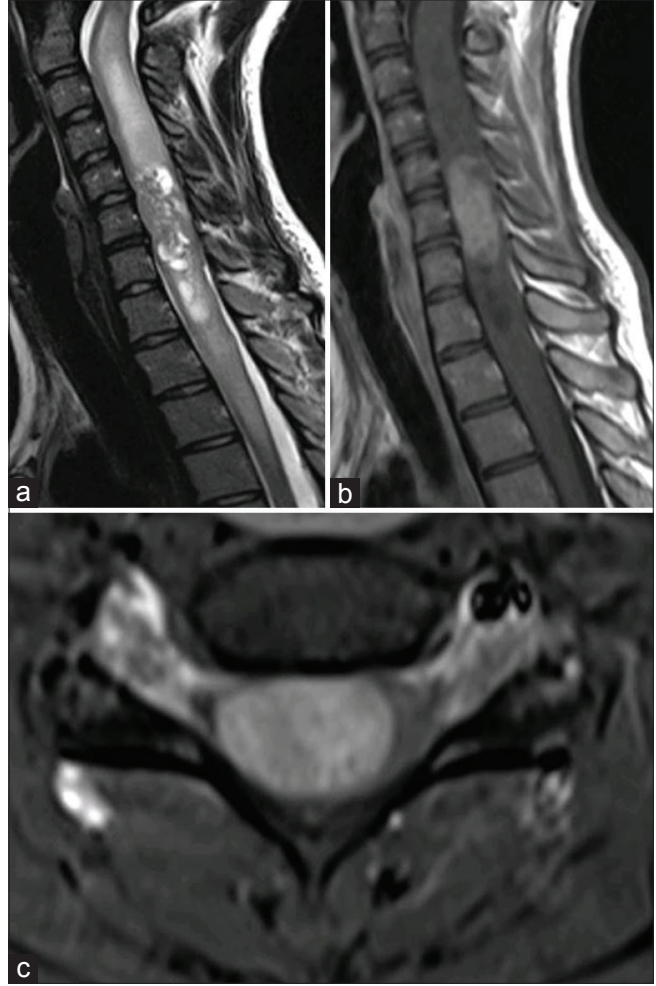

Figure 3: (a) MRI cervical spine T2 weighted sagittal image showing hyper-intense contrast enhancing lesion at C5-C7. (b) Magnetic resonance imaging (MRI) cervical spine TI weighted sagittal image showing hyperintense lesion at C5-C7. (c) MRI TI weighted axial image showing large circumscribed hyper-intense lesion pushing spinal cord to left side

In another study conducted in 2009, Matsuyama et al..$^{[12]}$ included 106 patients with a wide age range of 6-75 years. Their mean follow-up period was 7.3 years. They reported preoperative ambulatory status and GTR to be associated with better neurological prognosis; however, they observed postoperative neurological worsening in $31.5 \%$ patients. This was higher as compared to our observation of transient postoperative neurological deficits in 23.2\% patients. Only $11.6 \%$ of our patients developed permanent neurological deficits after surgery at last follow-up which is better than most than other studies that observed permanent deterioration in $11.6-34.6 \%$ patients. ${ }^{[2,10,12]}$

Management of tumors is always aimed at improving PFS. We have reported recurrence or disease progression in $18.6 \%$ patients, which is consistent with the recurrence rates of $9-22 \%$ in the current published literature. ${ }^{[2,10,18]}$ Earlier studies have reported high grade astrocytoma and malignant IMSCT along with extent of resection to be associated with increased risk of recurrence. ${ }^{[4,5]}$ In our study, more than half of the recurrences/disease progressions were seen in astrocytoma, however, it was not statistically significant $(P=0.308)$. We reported GTR and low grade of lesion to be significant predictors of PFS, which is in agreement with previous studies. ${ }^{[10,18]}$

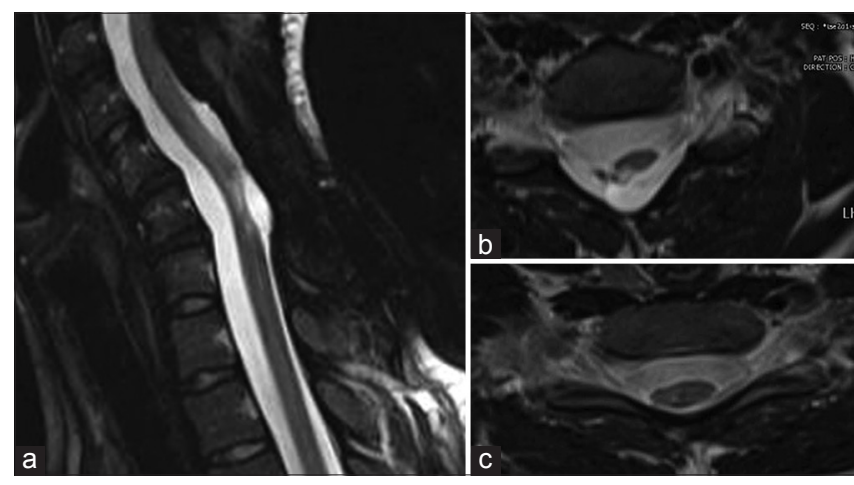

Figure 4: (a) Post-operative MRI cervical spine T2 weighted sagittal image showing thinning of spinal cord at C6. (b) Post-operative MRIT2 weighted axial image showing thin spinal cord surrounded by CSF. (c) MRIT2 weighted axial image showing normal section of spinal cord

There is controversy in existing literature regarding the role of adjuvant radiotherapy and chemotherapy for IMSCT, with some latest studies limiting its role to tumors which are not completely resectable. ${ }^{[3,7,18,19]}$ Approximately a quarter of our patients received adjuvant radiotherapy and none received chemotherapy, however, we did not find any significant impact of radiation therapy on PFS or neurological outcomes.

The use of intraoperative electrophysiology techniques including SSEP and/or MEP has been advocated to help maintain patient's neurological function after surgery, which along with excision of entire tumor is the aim in most procedures. In our study, IOEM was used in only $8(18.6 \%)$ patients, 3 of which had temporary and 1 had permanent postoperative worsening of neurological function. We did not observe any significant association of IOEM with extent of surgical resection or preservation of functional status. This is in contrast to studies at other centers which used this modality more often and attributed improved function due to its use improved outcomes. ${ }^{[6,9,12,17]}$ Our different findings may be due to less often use of this modality and technical difficulties.

We included patients operated by multiple surgeons at a single center and the follow-up period was small, which are important limitations of this study. Another important feature is the impact of different anatomical levels of disease on functional status, which was not found to be statistically significant in our study, which again might be due to the smaller number of study participants. However, considering the rarity of these tumors and insufficient data from developing countries, our study highlights important aspects in the presentation, management and outcomes of IMSCTs in our region.

\section{CONCLUSION}

We have achieved a GTR rate of $68.9 \%$ for IMSCTs using limited technological assistance. In few cases, 
where intraoperative electrophysiology was used, the rate of GTR was $100 \%$. Preoperative neurological status was associated with better postoperative McCormick score.

\section{Financial support and sponsorship \\ Nil.}

\section{Conflicts of interest}

There are no conflicts of interest.

\section{REFERENCES}

I. Bhatti SN, Khan SA, Raja RA, Shah R, Aurangzeb A, Khan AA, et al. Outcome of intramedullary spinal cord tumours: Experience with 18 patients operated at Ayub Teaching Hospital, Abbottabad. J Ayub Med Coll Abbottabad 2010;22:15-7.

2. Boström A, Kanther NC, Grote A, Boström J. Management and outcome in adult intramedullary spinal cord tumours: A 20-year single institution experience. BMC Res Notes 2014;7:I.

3. Chang UK, Choe WJ, Chung SK, Chung CK, Kim HJ. Surgical outcome and prognostic factors of spinal intramedullary ependymomas in adults. J Neurooncol 2002;57:133-9.

4. Cohen AR, Wisoff JH, Allen JC, Epstein F. Malignant astrocytomas of the spinal cord. J Neurosurg 1989;70:50-4

5. Constantini S, Miller DC, Allen JC, Rorke LB, Freed D, Epstein FJ. Radical excision of intramedullary spinal cord tumors: Surgical morbidity and long-term follow-up evaluation in 164 children and young adults. J Neurosurg 2000;93:I83-93.

6. Costa P, Bruno A, Bonzanino M, Massaro F, Caruso L, Vincenzo I, et al. Somatosensory-and motor-evoked potential monitoring during spine and spinal cord surgery. Spinal Cord 2007;45:86-9I.

7. Isaacson SR. Radiation therapy and the management of intramedullary spinal cord tumors. J Neurooncol 2000;47:231-8.

8. Klekamp J, Samii M. Surgery of spinal tumors. Berlin - Heidelberg: Springer; 2007.
9. Kothbauer KF, Deletis V, Epstein FJ. Motor-evoked potential monitoring for intramedullary spinal cord tumor surgery: Correlation of clinical and neurophysiological data in a series of 100 consecutive procedures. Neurosurg Focus 1998;4:E3.

10. Kumar R, Banerjee S. Management and functional outcome of intramedullary spinal cord tumors: A prospective clinical study. Asian J Neurosurg 2014;9:177.

II. Manzano G, Green BA, Vanni S, Levi AD. Contemporary management of adult intramedullary spinal tumors-Pathology and neurological outcomes related to surgical resection. Spinal Cord 2008;46:540-6.

12. Matsuyama Y, Sakai Y, Katayama Y, Imagama S, Ito Z, Wakao N, et al. Surgical results of intramedullary spinal cord tumor with spinal cord monitoring to guide extent of resection: Clinical article. J Neurosurg 2009; 10:404-13.

13. McCormick PC, Torres R, Post KD, Stein BM. Intramedullary ependymoma of the spinal cord. J Neurosurg 1990;72:523-32.

14. Morota N, Deletis V, Constantini S, Kofler M, Cohen H, Epstein FJ. The role of motor evoked potentials during surgery for intramedullary spinal cord tumors. Neurosurgery 1997;41:1327-36.

I5. Nakamura M, Ishii K, Watanabe K, Tsuji T, Takaishi H, Matsumoto M, et al. Surgical treatment of intramedullary spinal cord tumors: Prognosis and complications. Spinal Cord 2008;46:282-6.

16. Raco A, Esposito V, Lenzi J, Piccirilli M, Delfini R, Cantore G. Long-term follow-up of intramedullary spinal cord tumors: A series of 202 cases. Neurosurgery 2005;56:972-8I.

17. Sala F, Bricolo A, Faccioli F, Lanteri P, Gerosa M. Surgery for intramedullary spinal cord tumors: The role of intraoperative (neurophysiological) monitoring. Eur Spine J 2007; 16:130-9.

18. Sandalcioglu I, Gasser T, Asgari S, Lazorisak A, Engelhorn T, Egelhof T, et al. Functional outcome after surgical treatment of intramedullary spinal cord tumors: Experience with 78 patients. Spinal Cord 2005;43:34-4I.

19. Schwartz TH, McCormick PC. Intramedullary ependymomas: Clinical presentation, surgical treatment strategies and prognosis. J Neurooncol 2000;47:21 I-8.

20. Sevick RJ, Wallace CJ. MR imaging of neoplasms of the lumbar spine. Magn Reson Imaging Clin N Am 1999;7:539-53. 